\title{
INTELIGENCIA SEXUAL EN RELACIÓN CON LA AUTOESTIMA EN ADULTOS
}

\section{SEXUAL INTELLIGENCE IN RELATION TO SELF-ESTEEM IN ADULTS}

\author{
Estuardo Beethoven Paredes Morales ${ }^{1}$ \\ Ruth Stefanía Nacato Nato ${ }^{2}$ \\ Javier Alexander Salas Sandoval ${ }^{3}$
}

Recibido: 2020-01-05 / Revisado: 2020-04-15 / Aceptado: 2020-05-05 / Publicado: 2020-07-01

Forma sugerida de citar: Paredes-Morales, E. B., Ñacato-Ñato, R. S. y Salas-Sandoval, J. A. (2020). Inteligencia sexual en relación con la autoestima en adultos. Retos de la Ciencia. 4(9), pp. 37-45. https://doi.org/10.53877/rc.4.9.20200701.04

\section{RESUMEN}

La investigación tiene sus bases en la teoría del aprendizaje social y la cognición propuesta por Albert Bandura el objetivo principal es; hallar la relación entre inteligencia sexual y autoestima en personas adultas. La metodología utilizada fue de enfoque cuantitativo, de tipo no experimental, con un diseño transversal y un alcance correlacional asociativo; para ello se utilizó una muestra de tipo no probabilística por conveniencia de 384 personas adultas en la ciudad de Quito, Ecuador. Para la recolección de datos se recurrió a una encuesta sociodemográfica diseñada por los investigadores, el Test de inteligencia sexual de Sheree Conrad y Michael Milburn y la Escala de Autoestima de Rosemberg para adultos. Para la comprobación de la hipótesis se utilizó la prueba Chi-Cuadrado; cuyo valor $p=.000$, razón por la que se rechazó la hipótesis nula $(\mathrm{Ho})$, y se afirma que existe relación entre inteligencia sexual y autoestima. Con el fin de complementar dicha información se utilizó el coeficiente de correlación $V$ de Cramer cuyo resultado es $V=.263$ lo que significa que la relación existente entre las variables es débil. Por lo que se llega a la conclusión que a pesar de poseer una inteligencia sexual medianamente baja la auto estima es buena, además que la autoestima es mayormente buena de las personas de rangos de edades superiores a los 40 años en relación con grupos de edades más jóvenes.

Palabras clave: inteligencia sexual, autoestima, adultos.

\footnotetext{
${ }^{1}$ Psicólogo Clínico. Terapeuta Familiar. Profesional en Libre Ejercicio. Quito-Ecuador. E-mail: estuardoparedesmorales@yahoo.es / https://orcid.org/0000-0002-9722-5091

2Psicóloga Clínica. Profesional en Libre Ejercicio. Quito-Ecuador. E-mail: stefnia3@gmail.com / https://orcid.org/0000-0002-1055-0108

3Psicólogo Clínico. Profesional en Libre Ejercicio. Quito-Ecuador. E-mail: damonpsi@outlook.es / https://orcid.org/0000-0003-4896-4450
} 


\section{ABSTRACT}

The research is based on the theory of social learning and cognition proposed by Albert Bandura, the main objective is find the relationship between sexual intelligence and self-esteem in adults. The methodology used was of a quantitative approach, of a non-experimental type, with a cross-sectional design and an associative correlational scope; For this, a non-probabilistic convenience sample of 384 adults in the city of Quito, Ecuador was used. For data collection, a sociodemographic survey designed by the researchers was used, the Sheree Conrad and Michael Milburn Sexual Intelligence Test and the Rosemberg SelfEsteem Scale for adults. To test the hypothesis, the Chi-Square test was used; whose $\mathrm{p}$-value $=.000$, which is why the null hypothesis $(\mathrm{Ho})$ was rejected, and it is affirmed that there is a relationship between sexual intelligence and self-esteem. To complement this information, Cramer's $V$ correlation coefficient was used, the result of which is $V=.263$, which means that the relationship between the variables is weak. Therefore, it is concluded that despite having a moderately low sexual intelligence, self-esteem is good, besides, that self-esteem is mostly good for people with age ranges over 40 years regarding age groups older. young boys.

Keywords: sexual intelligence, self-esteem, adults.

\section{INTRODUCCIÓN}

Hablar de sexualidad conlleva comprender la complejidad de la naturaleza humana en la que existen también limitantes relacionados al desconocimiento y la banalización que están presentes dentro de una sociedad globalizada; considerando que los niños estadounidenses ocupan de seis a siete horas por día con alguna forma de medio masivo (Roberts, 2000). Además, que las influencias de los medios de comunicación de acuerdo con lo que establece (Gerbner et. al., 2002). Acerca del establecimiento de la agenda, cultivación y aprendizaje social a través de cine, novelas, música, van generando modelos o estereotipos basados en los estándares que el entorno contemporáneo pudiese considerar como deseables para el ciudadano en general, ya sea con respecto a la autoimagen tanto como al comportamiento sexual. Por esta razón y como objetivo principal se busca comprender la relación entre inteligencia sexual y autoestima en adultos, de modo tal que se comprenda dicha problemática a partir de la teoría que se aborda a continuación.

La base teórica elemental del aprendizaje a través de lo social y la cognición de Albert Bandura, nos permite explicar el desarrollo de cada una de las variables abordadas y además de este modo la cientificidad no será tácita sino argumentada, pues según Bandura (citado en Rivière, ano) el aprendizaje es: "el proceso por el cual se incorporan las normas sociales, costumbres e ideologías de una determinada sociedad" para complementar dicha mención, se destaca que... "el funcionamiento humano se explica teniendo en cuenta tres factores básicos entre los cuales están: la conducta, los factores personales cognitivos y los acontecimientos derivados del ambiente (Paredes, 2006, p. 201).

En base a lo mencionado, se infiere que parte del aprendizaje de los seres humanos ocurre en un entorno social. Además, el aprendizaje social y de la personalidad se basan en el principio de la "observación- imitación" según Bandura, lo cual significa que estos procesos van más allá del posicionamiento conductista 
que se basaba en refuerzos positivos y negativos, puesto que se comprende que la imitación de una conducta se puede dar en base a otras circunstancias porque son en su mayoría aprendidas y no innatas (citado en Paredes, 2006). Por ello es que se llegó a comprender que el aprendizaje por observación sucede a través de procesos intermedios entre estímulos y respuestas, entendiéndose a dichos procesos como cognición.

A mayor detalle, se rescata que la teoría del aprendizaje a través de lo social y la cognición; expone que el comportamiento se aprende a través de la observación, por medio de la cual se adquieren conocimientos, reglas, habilidades, estrategias, creencias y actitudes; por consiguiente, el aprendizaje se da por la asociación de los símbolos presentes en un ambiente como los estereotipos y comportamientos propios de una cultura y todo lo que a ella respecta y por lo tanto, es posible deducir cómo los procesos cognitivos de la persona y el medio interactúan entre sí para desarrollar comportamientos y conocimientos, para finalmente entenderse la construcción de la autoestima según los modelos que traducidos a un contexto determinado, se presentan como deseables o adecuados dependiendo donde se encuentre una persona pues se suscita el proceso de imitación u observación del modelo, refiriéndose a los estándares vigentes en la misma.

Durante este trabajo investigativo se menciona repetidamente a cada una de las variables abordadas, siendo estas: inteligencia sexual, autoestima y adultez. Por consiguiente y para comprender de mejor manera cada una de ellas, se ha hecho necesario elaborar una conceptualización referente a dichos temas.

La Inteligencia sexual que es la primera de las variables, término que fue descrito en el 2002, por Conrad y Milburn, quienes proponen un medio a través del cual se obtiene un coeficiente sexual y conocer hasta qué punto una persona está contenta con su vida sexual, en este sentido se refieren:

La capacidad de aprender a conocerse, valorar, liberarse del miedo y de la culpa, de ser capaces de informarse y aprender más acerca de la sexualidad, así como también descubrir que el sexo es mucho mejor cuando hay amor, apertura y respeto hacia la otra persona.

Por el contrario, existen personas limitadas en este aspecto pues solo pueden contribuir a su vida y a la de la pareja con relaciones tristes y pobres en toda la multiplicidad de aspectos positivos mencionados anteriormente y que la sexualidad conlleva. En consecuencia los estudios sobre Inteligencia Sexual, refieren que las personas que no han desarrollado este aspecto de acuerdo (Conrad y Milburn, 2002,) corren un mayor riesgo de caer en violencia sexual, sean estos los causantes o las víctimas, se pueden desencadenar conductas de promiscuidad o la adicción sexual, mismos que a la vez ocasionan prácticas sexuales riesgosas como las ITS, embarazos no deseados, incluso pueden llegar a perder el deseo sexual e incluso a prescindir totalmente de la sexualidad. Por ende, cuando las personas no le dan el suficiente valor a su sexualidad y no están dispuestas a aprender, perjudican considerablemente a la pareja, la imponen y condenan a unas carencias que en realidad se pueden mejorar y multiplicar. Por otra parte, los mismos autores Conrad y Milburn, refiere que la inteligencia sexual se compone de tres pilares fundamentales que son: consciencia del Yo sexual secreto, el conocimiento científico sobre sexualidad y la capacidad de conexión con otras personas. El primero de dichos pilares guarda relación con la siguiente variable a abordar, la autoestima pues Conrad y Milburn (2002) consideran que quienes son inteligentes sexualmente "comprenden su yo sexual secreto, el patrón de los deseos, 
necesidades, preferencias, temores incluso traumas, que guían su conducta sexual... son capaces de advertir cuando sus deseos sexuales están sustituyendo a carencias emocionales que no son sexuales" (p. 31).

En este apartado y con la finalidad de posibilitar la mayor comprensión del tema de manera concreta se analizará el concepto de la segunda variable autoestima. Etimológicamente la palabra autoestima, se forma por dos conceptos: "del griego autos, que se refiere a sí mismo y del latín estimare que significa valoración de la persona como tal, el aprecio de sí misma" (Lozano, Larrea, y Martínez 2017). Posteriormente se encuentra la propuesta de Branden, 2010 sobre autoestima mencionando que "Es una experiencia intima; y reside en el núcleo de nuestro ser. Es lo que yo siento y pienso sobre mí mismo" (p.18). Además, explica que la autoestima se vive como un; "juicio positivo sobre uno mismo, al haber conseguido un entramado personal coherente basado en los cuatro elementos básicos del ser humano: físicos, psicológicos, sociales y culturales. En estas condiciones va creciendo la propia satisfacción, así como la seguridad ante uno mismo y ante los demás". (Branden, 2010, p. 44)

Así mismo se encuentran aportes sobre autoestima realizado por Congost, 2014 quien expresa que; "es una experiencia subjetiva que nos condiciona a la hora de enfrentarnos con nuestro entorno. $Y$ lo hace porque interfiere directamente en nuestra relación con las demás personas" (p. 26). Considerando otras propuestas que aportan a la comprensión y construcción de una definición más puntual; menciona Lozano, Larrea, y Martínez que: "La autoestima tiene que ver con la aceptación de sí mismo, con quererse y aceptarse con sus cualidades, defectos y limitaciones, con la seguridad y confianza en sí mismo para salir adelante en la vida y afrontar retos (2017, p. 21).

En este sentido de acuerdo con los distintos autores abstrae que la autoestima es la percepción evaluativa propia de cada persona además del significado personal que todo individuo tiene de sí mismo. Las personas gozan de una forma particular de ser por la que se identifican, perciben y relacionan en este mundo aquello que caracteriza a cada individuo y la manera en que otros lo perciben.

La última variable considerada en la investigación respecta al grupo etáreo, la adultez, con el cual se trabajó, para poder establecer un rango más preciso de edad se consideró lo propuesto y que se, "Se denomina a la etapa entre los 20 y 65 años, pero esta definición es arbitraria” (p. 480). Además, también permite comprender la razón por la cual se consideró a la adultez como variable de estudio, al mencionar que con el transcurso de los años se experimentan "cambios tanto a nivel físico como psicológico que afectan al desarrollo y la identidad sexual de las personas" (Papalia, 2012, p. 430).

Por otra parte, esta etapa de la adultez involucra a la intimidad como un elemento vital, de forma que la sexualidad podría abarcar mayor profundidad y compromiso en las parejas, hecho que en otros ciclos vitales no se encuentra de manera muy frecuente como menciona (Paredes, 2006) en la adultez ya se han madurado los rasgos físicos y se ha afirmado la sexualidad, es decir, que cada sujeto se identifica y vive su sexualidad de la manera que mejor considere. Ahora a la medida que la adultez alcanza las distintas áreas o esferas de la vida como se menciona a priori, y considerando la idea de autoestima de Branden al indicar que "el modo en que nos sentimos con respecto a nosotros mismos afecta en forma decisiva todos los aspectos de nuestra experiencia, desde la manera en que funcionamos en el trabajo, el amor o el sexo" (2010, p. 1), se puede ver influenciado 
en alguna parte de su vida individual o interpersonal. De forma positiva o negativa según como se positiva esta realidad.

\section{METODOLOGÍA}

La metodología que se empleó fue de enfoque cuantitativo, de tipo no experimental, con un diseño transversal y un alcance correlacional asociativo (Sampieri, Fernández y Baptista (2014); para ello se obtuvo una muestra de tipo no probabilística por conveniencia de 384 personas adultas en la ciudad de Quito, Ecuador. Más adelante se realizó la recolección de datos de manera presencial y supervisada, a través de los siguientes instrumentos; el primero, una encuesta sociodemográfica diseñada por los investigadores, la prueba de inteligencia sexual de Sheree Conrad y Michael Milburn (2002) y la Escala de Autoestima de Rosemberg (1965) para adultos. Posteriormente para la comprobación de la hipótesis se utilizó la prueba Chi-Cuadrado; donde se trabajó con una significancia de $5 \%$ y se obtuvo el valor $p=.000$, razón por la que se rechazó la hipótesis nula $\left(\boldsymbol{H}_{\boldsymbol{o}}\right)$, en consecuencia, se afirma que existe relación entre inteligencia sexual y autoestima. Con el fin de complementar dicha información se utilizó el coeficiente de correlación V de Cramer, con un análisis estadístico que corresponde a V $=.263$

\section{RESULTADOS}

Tabla 1:

Inteligencia Sexual

\begin{tabular}{llllll}
\hline Nivel & Rangos & Frecuencia & Porcentaje & \multicolumn{2}{l}{ Rango de edad } \\
\cline { 4 - 6 } & & & & $20-40$ & $41-61$ \\
Muy bueno "A" & $>$ / = 90 pts. & 73 & $19,01 \%$ & $21.77 \%$ & $5.97 \%$ \\
Aceptable "B" & $80-89$ pts. & 57 & $14,84 \%$ & $14.83 \%$ & $14.93 \%$ \\
Media "C" & $70-79$ pts. & 85 & $22,14 \%$ & $24.29 \%$ & $11.94 \%$ \\
Bajo "D" & $60-69$ pts. & 57 & $14,84 \%$ & $12.30 \%$ & $26.87 \%$ \\
Muy bajo "E" & $<60$ pts. & 112 & $29,17 \%$ & $26.81 \%$ & $40.30 \%$ \\
Total & & 384 & $100 \%$ & $100 \%$ & $100 \%$ \\
\hline
\end{tabular}

Fuente: Instrumentos aplicados

Elaborado por: Los autores

De acuerdo con el análisis de los resultados estadísticos, en inteligencia sexual, se determina que existe una partición de porcentajes entre un nivel muy bueno a muy bajo, existiendo saltos categóricos con referencia a los rangos de edad, constituyendo entre el $28,87 \%$ en bajo y el $40,30 \%$ en muy bajo el nivel de Inteligencia sexual lo que llama la atención en vista que existe un $22,14 \%$ de un grupo de edad de 20-40 años en su inteligencia sexual. 
Tabla 2:

Autoestima

Nivel

Rangos

Frecuencia

Porcentaje

Rango de edad

20-40

41-61

Elevada

$30-40$ pts. 251

$65,36 \%$

$61.20 \%$

$84.07 \%$

Media

$26-29$ pts. $\quad 80$

$20,83 \%$

$22.08 \%$

$14.93 \%$

Baja

$<25$ pts.

53

384
$13.80 \%$

$100 \%$
$16.72 \% \quad 0 \%$

$100 \% \quad 100 \%$

Fuente: Instrumentos aplicados

Elaborado por: Los autores

De acuerdo con el análisis de los resultados estadísticos, en auto estima, se determina que el $65,36 \%$, de las personas tienen una auto estima elevado, además de ser el rango de edad 41-61 años, a los que más puntúan y en esta edad nadie tiene baja autoestima. Se puede considerar como personas que alcanzaron una madurez psicológica.

Tabla 3:

Nivel de inteligencia sexual y el nivel de autoestima

\begin{tabular}{|c|c|c|c|c|c|c|}
\hline & & & \multicolumn{3}{|c|}{ AUTOESTIMA } & \multirow{2}{*}{$\begin{array}{l}\text { Total, } \\
\text { genera }\end{array}$} \\
\hline & & & ELEVADA & MEDIA & BAJA & \\
\hline \multirow{12}{*}{ 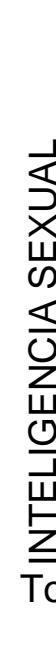 } & MUY BUENO & Frecuencia & 66 & 6 & 1 & 73 \\
\hline & & $\%$ & $17.2 \%$ & $1.6 \%$ & $0.3 \%$ & $19.0 \%$ \\
\hline & ACEPTABLE & Frecuencia & 38 & 13 & 6 & 57 \\
\hline & & $\%$ & $9.9 \%$ & $3.4 \%$ & $1.6 \%$ & $14.8 \%$ \\
\hline & MEDIA & Frecuencia & 55 & 9 & 21 & 85 \\
\hline & & $\%$ & $14.3 \%$ & $2.3 \%$ & $5.5 \%$ & $22.1 \%$ \\
\hline & BAJO & Frecuencia & 38 & 10 & 9 & 57 \\
\hline & & $\%$ & $9.9 \%$ & $2.6 \%$ & $2.3 \%$ & $14.8 \%$ \\
\hline & MUY BAJO & Frecuencia & 54 & 42 & 16 & 112 \\
\hline & & $\%$ & $14.1 \%$ & $10.9 \%$ & $4.2 \%$ & $29.2 \%$ \\
\hline & yeneral & Frecuencia & 251 & 80 & 53 & 384 \\
\hline & & $\%$ & $65.4 \%$ & $20.8 \%$ & $13.8 \%$ & $100 \%$ \\
\hline
\end{tabular}

Fuente: Instrumentos aplicados

Elaborado por: Los autores

De acuerdo con el análisis de los resultados estadísticos, auto estima e inteligencia sexual se determina que la autoestima es elevada $65.4 \%$, mientras la inteligencia sexual se mantiene una media entre medio bajo $22.1 \%$ y muy bajo $29.2 \%$, esto hace pensar que podemos tener una buena autoestima con un promedio bajo de inteligencia sexual.

A través de la encuesta sociodemográfica se encontró que la autoestima según la variable sexo, donde las personas adultas puntuaron autoestima elevada, 
calificando significativamente con porcentajes de $65,75 \%$ para hombres y el $65,13 \%$ para mujeres.

Además con la encuesta sociodemográfica se pudo constatar que los porcentajes más representativos de aquellas personas que leyeron 3 o más libros son quienes presentan una elevada autoestima con un $25.50 \%$, mientras que aquellos que no han leído ningún libro o artículo sobre sexualidad, presentaron un nivel medio de autoestima con un $41,56 \%$, finalmente todas las personas adultas que presentan baja autoestima, el mayor porcentaje pertenece a aquellos que no han leído ningún libro sobre sexualidad con un $39.29 \%$.

\section{DISCUSIÓN}

mayor porcentaje pertenece a aquellos que no han leído ningún libro sobre sexualidad con un $39.29 \%$. Vale traer a colación lo mencionado en la revisión de la literatura por Para empezar esta sección es menester retomar los datos obtenidos en base a los resultados de la investigación, donde se puede observar que el $29.17 \%$ del total de la muestra de 384 personas adultas, presentan un nivel muy bajo de inteligencia sexual, estos resultados presenta relación con los resultados de un estudio realizado por Paredes, et al. (2018) sobre la Inteligencia sexual en hombres y mujeres ecuatorianos en relación conyugal donde se encontró que la inteligencia sexual es relativamente baja en el ecuatoriano en general con $72,96 \%$ de una muestra de 312 personas adultas. Datos que también vale comparar con los resultados obtenidos en la investigación de Rosero (2017), donde se encontró que el $75 \%$ de la muestra se encuentra entre el nivel medio y muy bajo. El resultado obtenido puede ser atribuido al desarrollo insuficiente o nulo de uno de los pilares de la inteligencia sexual que es los conocimientos científicos sobre sexualidad principalmente, pues permite desmitificar a la sexualidad y ampliar la información que se tiene respecto a la misma.

Por medio de los resultados también se evidenció que la mayoría de personas que obtuvieron un nivel de autoestima elevada, también obtuvieron el nivel de inteligencia sexual muy alto con un porcentaje correspondiente al 17,2\%, reforzándose la conclusión de Herrero,1994, quien menciono que la autoestima influye directamente en el comportamiento de los individuos e incide en la promoción del bienestar psicológico, pues la forma en que se evalúa a sí mismo el individuo tiene repercusión en todas las áreas del desarrollo social, emocional, intelectual, conductual y escolar (como se citó en González, Valdés y Serrano, 2003). De manera que se insiste en la importancia de la autoestima en las distintas esferas del ser humano, en el caso de la presente investigación, en la esfera sexual.

En cuanto a la autoestima, se encontró que el $65,36 \%$ del total de la muestra de personas adultas presentaron autoestima elevada, en contraste a la investigación: "La funcionalidad familiar asociada al desempeño sexual, y la autoestima en adultos varones", donde la autoestima regular se presentó con mayor frecuencia, con un porcentaje del 33.9\% (Pérez 2014) donde resalta que el entorno familiar influye sobre el desarrollo del nivel de autoestima.

Además se halló que la autoestima según la variable sexo, los hombres y las mujeres obtuvieron puntajes similares, sin embargo la diferencia es ligeramente significativa siendo los hombres quienes presentan un puntaje mayor al de las mujeres, lo cual se corrobora en la investigación de García (2016) presenta resultados en la revista: Journal of Personality and Social Psychology, donde explica 
que los hombres tienen mejor opinión de sí mismos que las mujeres en prácticamente todas las culturas del mundo y todos los tramos de edad, por lo tanto, los hombres demuestran mejor autoestima, dato que en la presente investigación se observa aunque no de manera tan significativa. Cabe recalcar que la presente investigación tuvo como limitante el poseer una muestra heterogénea, es decir, que la mayor cantidad de participantes fueron mujeres, razón por la cual sin una adecuada homologación los resultados posiblemente no reflejarán la información más precisa.

Finalmente, a través de los resultados en relación al número de libros leídos sobre temas de sexualidad y cómo esto interviene en la autoestima, con el cual se realizó parangón por lo mencionado por Conrad y Milburn (2002) "Tener información incrementa la confianza en nosotros mismos y la autoestima" (p.30). Lo que se pudo observar en los resultados de la presente investigación fue que aquellas personas que leyeron 3 o más libros, presentan una autoestima elevada con un $25.50 \%$, mientras que aquellos que no han leído ningún libro o artículo sobre sexualidad, presentaron un nivel de autoestima media con un $41,56 \%$, finalmente todas las personas adultas que presentan autoestima baja, el Branden (2010), respecto a la autoestima pues "el modo en que nos sentimos con respecto a nosotros mismos afecta en forma decisiva todos los aspectos de nuestra experiencia". Se pone en evidencia y se demuestra que las personas con mayor autoestima están más dispuestas y se infiere también que posiblemente más motivadas a adquirir conocimientos.

\section{CONCLUSIONES}

A través de la estadística en la investigación se encontró que el valor de $p=$ .000 , en tal virtud se afirma que existe relación entre inteligencia sexual y autoestima, sin embargo gracias al coeficiente de correlación $\mathrm{V}$ de Cramer con el fin de complementar dicha información, se obtuvo que $\mathrm{V}=.263$ lo que significa que la relación existente entre inteligencia sexual y autoestima es "débil", donde la mayor cantidad de personas que obtuvieron un nivel muy bueno de inteligencia sexual también obtuvieron un nivel elevado de autoestima, mientras que en los niveles medio y bajo no existe dicha relación.

Con respecto a los resultados obtenidos con la prueba de inteligencia sexual, los adultos presentan en su mayoría un nivel de inteligencia sexual muy bajo que corresponde al valor categórico de $\mathrm{E}$, es decir el $29,17 \%$ de la muestra con puntajes inferiores a 60 puntos.

En cuanto a los resultados obtenidos de la prueba de autoestima de Rosemberg, la mayor cantidad de adultos evaluados presentan un nivel de autoestima elevado referentes al 65,3\% que corresponde a puntajes de 30 a 40 puntos. En este sentido la inteligencia sexual al parecer no influye mayormente en la autoestima, ahora se puede considerar por ser un tema nuevo dentro de la conducta sexual humana.

\section{REFERENCIAS}

Ahumada, S. Lüttges, C. Molina, T. y Torres, S. (2014). Satisfacción sexual: revisión de los factores individuales y de pareja relacionados. Revista Hospitalaria Clínica Universidad de Chile. 
Amezúa, E. (2004). Reflexiones y propuestas de modificación. Anuario de Sexología.

Branden, N. (2010). Cómo mejorar su autoestima. Grupo Planeta (GBS).

Congost, S. (2015). Autoestima automática. Editorial Planeta, S. A.

Conrad, S. y Milburn, M. (2002). Inteligencia sexual. Crown Publishers.

Gómez, J. (2013). Psicología de la sexualidad. Alianza Ed, SA., Madrid.

Gerbner, George, Gross, L., \& Morgan, M. (2002). Growing up with television: Cultivation processes. In J. Bryant \& D. Zill-man (Eds.), Media effects: Advances in theory and research: (2nd ed., pp. 43-67). Mahwah, NJ: Erlbaum.

González, N. y López, A. (2001). La autoestima: Colección Ciencias de la salud: Serie Ciencias de la conducta. México: UAEM.

Johnson, R. y Kuby, P. (2012). Estadística Elemental 11a edición. Cengage Learning.

Jurgenson, J. (2011). Sexo Terapia Integral. Segunda edición. Editorial El Manual Moderno S.A.

Kendall, M y Buckland, W. (1980). Diccionario de estadística (Dictionary of Statistical Terms). Ediciones Pirámide S.A.

Lozano, E. Larrea, G. y Martínez, R. (2017). Autoestima e Inteligencia Emocional. Ediciones. Ecuafuturo. 21p.

Morris, C. 2011. Introducción a la Psicología. Pearson educadión.

Papalia, D. Feldman, R. y Martorell, G. (2012). Desarrollo humano. McGraw-Hill. Paredes, A. (2006). Compendio de Psicología. San Marcos.

Paredes, E. B., Polanski, T. X., Porras, R. M., \& Gamboa, J. (2018). Inteligencia sexual en hombres y mujeres ecuatorianos en relación conyugal. PSIENCIA: Revista Latinoamericana de Ciencia Psicológica, 10(1), 2.

Ramos, C. (2004). Manual de métodos y técnicas de investigación en ciencias del comportamiento. Biblioteca Nueva, S.L.

Roberts, D. (2000). Media and youth: Access, exposure, and privatization.

Journal of Adolescent Health, 27 (2), 8-14.

Sampieri, C. (2014). Metodología de la investigación. Panamericana Formas e Impresos SA.

Shibley, J. y Delamater, J. (2006). Sexualidad humana. Editorial McGraw Hill.

Spiegel, M. y Stephens, L. (1970). Estadística-Serie Schaum. Mc Graw-Hill. 